\title{
Perturbation Theory in Nonstandard Cosmology
}

\author{
Günter Scharf \\ Physics Institute, University of Zürich, Winterthurerstr. 190 , CH-8057 Zürich, Switzerland \\ Email: scharf@physik.uzh.ch
}

\begin{abstract}
We explore a nonstandard model for the Universe in which we take a vacuum solution of Einstein's equation as background and introduce visible matter and radiation as perturbations. The perturbation theory is greatly simplified by using the mapping on the inner Schwarzschild solution. The resulting linear perturbation equations can be solved by power series. The solution leads to a finite energy density, but the pressure must be zero. A nonzero pressure comes in the exceptional case of spherical perturbation which gives the isotropic CMB perturbation. The model needs further exploration, in particular a quantitative treatment of CMB, to determine if it is fully consistent with our Universe.
\end{abstract}

Keywords: Cosmology

\section{Introduction}

The isotropy of CMB clearly shows that the cosmic gravitational field is spherically symmetric up to small anisotropies. However the visible matter is obviously anisotropic, furthermore its density is small compared to the critical density. In this situation a realistic description should start from a spherically symmetric vacuum solution of Einstein's equation, followed by the analysis of its anisotropic perturbations. The question then is, which vacuum solution should be chosen as background. This problem was solved in a previous paper [1]. If one assumes a one-to-one correspondence between comoving coordinates and the cosmic rest frame, then there is essentially only one spherically symmetric solution of Einstein's equations simultaneously in both systems. It is the homogeneous Datt or Kantowski-Sachs solution ([3], p.110) which in comoving coordinates has a line element of the form

$$
d s^{2}=d t^{2}-X(t)^{2} d r^{2}-Y(t)^{2}\left(d \vartheta^{2}+\sin ^{2} \vartheta d \phi^{2}\right) .
$$

Needless to say that we prefer the +--- metric because considering spin 2 gauge theories on the same basis as spin 1 [2], we cannot accept equations as $p^{2}=-m^{2}$ which follow with the signature -+++ . In contrast to standard FLRW cosmology there is no factor $r^{2}$ in front of $Y(t)$, therefore we call the metric (1.1) strongly homogeneous, because there is no $r$-dependence in the metric functions.

There is some confusion in the literature about the use of the notions "homogeneous" and "isotropic". For example Kantowski-Sachs call the solution (1.1) anisotropic although it is spherically symmetric and the angular variables can be separated in the perturbation theory. The notion "homogeneous" too has various different meanings [3]. In addition, it is difficult to select the correct name for (1.1) from the authors Schwarzschild, Lemaître, Datt, Ruban, Kantowski-Sachs etc. So we shall simply say nonstandard background in the following.

In the standard FLRW model one needs high-density hypothetical sources of the gravitational field, dark matter, dark energy or cosmological constant and an inflaton field, in order to get agreement with observations. Since none of these sources has been directly observed, non-standard cosmology based on (1.1) starts without sources from a vacuum solution which is consistent with the magnitude-redshift data. In fact it was shown in [1] sect.7 and is rederived in the next section that one can get an excellent representation of the current Hubble data by means of the vacuum solution corresponding to (1.1). Matter and radiation are considered as perturbations on the nonstandard background (1.1). The necessary perturbation theory is developed in this paper.

It is known ([3], p.386) that the nonstandard solution can be mapped on the inner Schwarzschild solution by exchanging time with the radial coordinate $r$. The mapping then is a consequence of Birkhoff's theorem. Do we really live inside of a black hole ? Not at all. The mapping is a purely mathematical 
operation by means of suitable (Schwarzschild) coordinates which have no physical meaning in the cosmological context. For the physical interpretation of results and comparison with observations one must always transform back to the comoving coordinates used in (1.1) or to the universal cosmic rest frame [1]. But to solve the equations of perturbation theory the mapping to the Schwarzschild solution is very useful. Frank J. Zerilli has developed the perturbation theory of the outer Schwarzschild solution in his Ph.D thesis of 1969 [4]. With the appropriate sign changes his equations can be taken over to our situation. Then transforming back to comoving coordinates gives us the results for our Universe.

The paper is organized as follows. In the next section we introduce the standard background and derive its magnitude-distance relation. We have one free parameter beside the Hubble constant. Using the measured value at $z=1$ we get an excellent representation of the entire Hubble diagram between $0<z<10$. In sect.3 we discuss the mapping between the nonstandard background and the inner Schwarzschild solution. In sect. 4 this correspondence is used to obtain the equations of first order perturbation theory. This is a direct application of the $\mathrm{PhD}$ thesis of F.J.Zerilli [4]. Zerilli has reduced the resulting linear differential equations to a wave equation of Schrödinger type which, however, cannot be solved analytically. Instead we transform in sect.5 to appropriate coordinates which are linearly related to the redshift. Then the perturbative equations are solved by power series. In the last section we briefly discuss the possible applications of the results in cosmology. In particular we comment on the excluded spherical case $L=0$ which leads to CMB.

\section{Nonstandard Background and Its Hubble Diagram}

Instead of using Bondi's symbol $Y(t)$ in (1.1) [5], we shall now write

$$
d s^{2}=d t^{2}-X(t)^{2} d r^{2}-R(t)^{2}\left(d \vartheta^{2}+\sin ^{2} \vartheta d \phi^{2}\right)
$$

because we need the symbol $Y(\vartheta, \phi)$ for the spherical harmonics. A second reason for this is that we are going to consider $R(t)$ as a new (radial !) coordinate. In [1] we have found the following parametric representation for $R(t)$ and $X(t)$ (equ.(5.14) and (5.18))

$$
\begin{gathered}
R(t)=T_{L} \sin ^{2} w, \\
X(t)=|\cot w|
\end{gathered}
$$

where the parameter $w$ varies between $\pi / 2$ and $\pi$. The comoving time is given by (equ.(5.8) in [1])

$$
t=T_{L}(w-\sin w \cos w)
$$

here $T_{L}$ determines the lifetime of the Universe. In addition we know from [1] equ.(5.25) that $X(t)$ is proportional to

$$
\dot{R}(t)=2 T_{L} \sin w \cos w \frac{d w}{d t} .
$$

Substituting

herein we obtain

$$
\frac{d t}{d w}=2 T_{L} \sin ^{2} w
$$

$$
\dot{R}(t)=\frac{\cos w}{\sin w}=\cot w=-X .
$$

For the physical discussion we need the radial null geodesics given by the wave vector $k^{\mu}=\left(1 / X,-1 / X^{2}, 0,0\right)$. Then the redshift is equal to

$$
1+z=\frac{X_{\mathrm{obs}}}{X_{\mathrm{em}}}=\frac{\cot w_{\mathrm{obs}}}{\cot w_{\mathrm{em}}}
$$

where em and obs refer to the time of emission and observation, respectively. The Big Bang corresponds to $z=\infty$, that means $w_{\mathrm{em}}=\pi / 2$, and $\pi / 2<w_{\mathrm{obs}}<\pi$, because $z(2.8)$ must be positive. From

$$
d z=\frac{\cot w_{\mathrm{obs}}}{\cot ^{2} w} \frac{d w}{\sin ^{2} w}=\frac{\cot w_{\mathrm{obs}}}{\cos ^{2} w} \frac{d t}{2 T_{L} \sin ^{2} w}
$$


we identify the Hubble constant

$$
\left.\frac{d z}{d t}\right|_{z=0}=-H_{0}=\frac{\left(1+\cot ^{2} w_{\mathrm{obs}}\right)^{2}}{2 T_{L} \cot w_{\mathrm{obs}}} .
$$

To calculate the radial distance we integrate

$$
\frac{d r}{d z}=\frac{d r}{d t} \frac{d t}{d z}=\frac{2 T_{L} \cot ^{2} w}{X \cot w_{\mathrm{obs}}}=\frac{2 T_{L}(1+z)^{3}}{\left[(1+z)^{2}+\cot ^{2} w_{\mathrm{obs}}\right]^{2}}
$$

from $z=0$ to $z>0$. With the new variable of integration $x=1 /(1+z)$ we get

$$
r(z)=2 T_{L} \int_{1 /(1+z)}^{1} \frac{d x}{x\left(1+\cot ^{2} w_{\mathrm{obs}} x^{2}\right)^{2}} .
$$

We introduce the parameter

$$
\alpha=\frac{1}{\left|\cot w_{\mathrm{obs}}\right|}
$$

which is also equal to the local light speed $c_{0}=d r / d t=1 /|X|$. Using the Hubble constant $(2.10)$ we finally obtain

$$
r(z)=\frac{c_{0}}{H_{0}}\left(1+\alpha^{2}\right)^{2} \int_{1 /(1+z)}^{1} \frac{d x}{x\left(\alpha^{2}+x^{2}\right)^{2}} .
$$

The luminosity distance is equal to $(1+z) r(z)$. The rational integral in $(2.11)$ is elementary so that

$$
\begin{gathered}
d_{L}(z)=\frac{c_{0}}{H_{0}}(1+z) \frac{\left(1+\alpha^{2}\right)^{2}}{2 \alpha^{2}}\left[\frac{1}{1+\alpha^{2}}-\frac{(1+z)^{2}}{1+\alpha^{2}(1+z)^{2}}+\right. \\
\left.+\frac{1}{\alpha^{2}} \log \frac{1+\alpha^{2}(1+z)^{2}}{1+\alpha^{2}}\right]
\end{gathered}
$$

The magnitude $m(z)$ is defined by

$$
m(z)=5 \log _{10} d_{L}+M+25
$$

where $M$ is the absolute magnitude of the supernova standard candle. In the Hubble diagram one plots the distance modulus

$$
\mu(z)=m(z)-M
$$

The parameter $\alpha^{2}$ will now be determined by the Hubble data. The measured Hubble diagram is nicely represented by the standard FLRW luminosity distance

$$
\tilde{d}_{L}(z)=\frac{c_{0}(1+z)}{H_{0}} \int_{1}^{1+z} \frac{d x}{\sqrt{\Omega_{M} x^{3}+\Omega_{\Lambda}}} .
$$

From the type Ia supernovae observations one has obtained the following parameter values $\Omega_{M}=0.27$, $\Omega_{\Lambda}=0.73, H_{0}=72 \mathrm{~km} /(\mathrm{s} \mathrm{Mpc})$. This is the best fit in [9]. In the table the corresponding distance modulus $\tilde{\mu}(z)$ is listed in the second column. We have taken the value $\tilde{\mu}(1)=44.08$ at $z=1$ as a measured value and have determined the free parameter $\alpha^{2}$ in (2.14) such that this value is reproduced. The result is

$$
\alpha^{2}=6.71
$$

With this value the entire Hubble diagram until $z=10$ is excellently represented by (2.14-16) as can be seen in the third column of the table. The last two columns show the look-back times in the standard and nonstandard models [1]. 


\begin{tabular}{|c|c|c|c|c|}
\hline$z$ & $\tilde{\mu}(z)(\mathrm{mag})$ & $\mu(z)(\mathrm{mag})$ & $\tilde{t}(z)\left(10^{9} Y\right)$ & $t(z)\left(10^{9} Y\right)$ \\
\hline 0.01 & 33.12 & 33.12 & 0.1349 & 0.1348 \\
\hline 0.02 & 34.64 & 34.64 & 0.2678 & 0.2676 \\
\hline 0.03 & 35.53 & 35.54 & 0.3990 & 0.3985 \\
\hline 0.04 & 36.17 & 36.18 & 0.5283 & 0.5275 \\
\hline 0.05 & 36.67 & 36.68 & 0.6558 & 0.6546 \\
\hline 0.06 & 37.08 & 37.09 & 0.7816 & 0.7799 \\
\hline 0.07 & 37.43 & 37.44 & 0.9057 & 0.9034 \\
\hline 0.08 & 37.74 & 37.75 & 1.0281 & 1.025 \\
\hline 0.09 & 38.01 & 38.02 & 1.1488 & 1.145 \\
\hline 0.1 & 38.25 & 38.26 & 1.2679 & 1.263 \\
\hline 0.2 & 39.89 & 39.91 & 2.3756 & 2.360 \\
\hline 0.3 & 40.89 & 40.91 & 3.3443 & 3.317 \\
\hline 0.4 & 41.62 & 41.64 & 4.1969 & 4.158 \\
\hline 0.5 & 42.20 & 42.22 & 4.9489 & 4.903 \\
\hline 0.6 & 42.69 & 42.71 & 5.6145 & 5.565 \\
\hline 0.7 & 43.10 & 43.12 & 6.2054 & 6.157 \\
\hline 0.8 & 43.46 & 43.48 & 6.7317 & 6.690 \\
\hline 0.9 & 43.79 & 43.80 & 7.2020 & 7.171 \\
\hline 1.0 & 44.08 & 44.08 & 7.0236 & 7.608 \\
\hline 2.0 & 46.05 & 45.96 & 10.181 & 10.45 \\
\hline 3.0 & 47.22 & 47.03 & 11.318 & 11.90 \\
\hline 4.0 & 48.05 & 47.78 & 11.928 & 12.79 \\
\hline 5.0 & 48.70 & 48.36 & 12.300 & 13.38 \\
\hline 6.0 & 49.22 & 48.82 & 12.541 & 13.80 \\
\hline 7.0 & 49.67 & 49.21 & 12.711 & 14.12 \\
\hline 8.0 & 50.05 & 49.55 & 12.836 & 14.37 \\
\hline 9.0 & 50.38 & 49.84 & 12.93 & 14.57 \\
\hline 10.0 & 50.68 & 50.10 & 13.033 & 14.73 \\
\hline
\end{tabular}

\section{Mapping on the Inner Schwarzschild Solution}

To map the nonstandard background to the Schwarzschild solution we choose $R$ as a new coordinate. Then we have

$$
\sin w=\sqrt{\frac{R}{T_{L}}}
$$

so that

$$
X^{2}=Q^{2} \frac{T_{L}-R}{R}
$$

and

$$
d t=2 T_{L} \sin ^{2} w d w=2 R d w=\tan w d R .
$$

Then $d s^{2}(2.1)$ assumes the following form

$$
d s^{2}=\left(\frac{R}{T_{L}-R}\right) d R^{2}-Q^{2} \frac{T_{L}-R}{R} d r^{2}-R^{2}\left(d \vartheta^{2}+\sin ^{2} \vartheta d \phi^{2}\right) .
$$

The integration constant $Q$ was not fixed in (2.7). Now we must take $Q^{2}=1$ to have a solution of Einstein's equations and this is the inner Schwarzschild solution because $R<T_{L}$ by $(2.2)$. However the comoving radial coordinate $r$ now sits at the place of the time coordinate, we shall write

$$
S=r
$$


in the following, so that $(S, R)$ are our inner Schwarzschild coordinates:

$$
d s^{2}=g_{\mu \nu}^{0} d x^{\mu} d x^{\nu}=\left(\frac{R}{T_{L}-R}\right) d R^{2}-\frac{T_{L}-R}{R} d S^{2}-R^{2}\left(d \vartheta^{2}+\sin ^{2} \vartheta d \phi^{2}\right) .
$$

This is our background metric in the new coordinates. The components of the Jacobian are equal to

$$
\frac{\partial t}{\partial R}=-\sqrt{\frac{R}{T_{L}-R}}, \quad \frac{\partial r}{\partial S}=1
$$

and otherwise zero. The minus sign follows from (2.6) because $\pi / 2<w<\pi$. Our lifetime constant $T_{L}$ is equal to $2 m$ where $m$ is the mass of the black hole. The Big Bang $w=\pi / 2$ corresponds to the horizon $R=T_{L}$ whereas $w=\pi$ is the true singularity $R=0$ of the Schwarzschild solution.

Since the Schwarzschild background (3.4) is spherically symmetric, the first order perturbation $h_{\mu \nu}(S, R, \vartheta, \phi)$ is expanded in tensor harmonics. The most explicit expressions for the 10 harmonics are given by Zerilli [4] in his Appendix A. Only the notation of these harmonics has been changed in later work [6]. For this reason we give the connection between Zerilli's notation (in brackets) and the one of Gerlach and Sengupta [6]:

$$
\begin{gathered}
\left(a_{L M}^{(0)}, a_{L M}^{(1)}, a_{L M}\right)=Y(\vartheta, \phi) \\
\left(b_{L M}^{(0)}, b_{L M}\right)=Y,_{a} \\
\left(c_{L M}^{(0)}, c_{L M}\right)=S_{a} \\
\left(d_{L M}\right)=S_{a ; b} \\
\left(g_{L M}\right)=Y \gamma_{a b} \\
\left(f_{L M}\right)=Y,_{a ; b} .
\end{gathered}
$$

The harmonics (3.8-9) have odd parity and are called axial or magnetic, the other seven have even parity and are called polar or electric. These all are $4 \times 4$ symmetric tensors which are orthonormal on the 2 -sphere. For later use we write down the following four harmonics

$$
\begin{gathered}
a_{L M}^{(0)}=\left(\begin{array}{cccc}
Y_{L M} & 0 & 0 & 0 \\
0 & 0 & 0 & 0 \\
0 & 0 & 0 & 0 \\
0 & 0 & 0 & 0
\end{array}\right) \\
a_{L M}^{(1)}=\frac{i}{\sqrt{2}}\left(\begin{array}{cccc}
0 & Y_{L M} & 0 & 0 \\
Y_{L M} & 0 & 0 & 0 \\
0 & 0 & 0 & 0 \\
0 & 0 & 0 & 0
\end{array}\right)
\end{gathered}
$$

As energy-momentum tensor we consider a perfect fluid with

$$
t_{\mu \nu}=(\varrho+p) u_{\mu} u_{\nu}-p g_{\mu \nu}
$$

where $\varrho$ and $p$ are density and pressure of the matter and $u_{\mu}$ its 4 -velocity, the latter is $u_{\mu}^{0}=(1,0,0,0)$ in comoving coordinates. The first order perturbation of the vacuum is given by

$$
\delta t_{\mu \nu}=(\delta \varrho+\delta p) u_{\mu}^{0} u_{\nu}^{0}-\delta p \bar{g}_{\mu \nu}^{0}
$$

where $\bar{g}_{\mu \nu}^{0}$ is the background (2.1) in comoving coordinates. We have the following diagonal tensor:

$$
\delta t_{\mu \nu}=\operatorname{diag}\left(\delta \varrho, \delta p X^{2}(t), R^{2}(t) \delta p, R^{2} \sin ^{2} \vartheta \delta p\right) .
$$

This tensor must also be transformed into the Schwarzschild coordinates according to

$$
\delta T_{\alpha \beta}=\delta t_{\mu \nu} \frac{\partial \bar{x}^{\mu}}{\partial \alpha} \frac{\partial \bar{x}^{\nu}}{\partial \beta}
$$


where the bar-coordinates are the comoving ones $(t, r)$. Using the values of the derivatives (3.5) we obtain

$$
\delta T_{\alpha \beta}=\operatorname{diag}\left(\delta p, \delta \varrho \frac{R}{T_{L}-R}, R^{2} \delta p, R^{2} \sin ^{2} \vartheta \delta p\right)
$$

It is essential to notice that density and pressure perturbations are interchanged as a consequence of (3.5). The diagonal form shows that only the tensor harmonics $a_{L M}^{(0)}, a_{L M}$ and $g_{L M}$ in (3.12-13) contribute in the perturbation theory next section.

\section{Zerilli's Perturbation Theory Applied to the Inner Schwarzschild Solution}

In this section we always calculate with Schwarzschild coordinates inside the horizon. The metric perturbations $\delta g_{\mu \nu}$ are usually denoted by $h_{\mu \nu}$. If $G_{\mu \nu}$ is the Einstein tensor we have to solve the perturbed Einstein equation

$$
\delta G_{\mu \nu}=8 \pi G \delta T_{\mu \nu}
$$

where $G$ is Newton's constant. Expanding the perturbations and the right side of (4.1) in tensor harmonics, the angular variables $(\vartheta, \phi)$ in the linear equation (4.1) are separated and we are left with 10 linear partial differential equations in $(S, R)$. The 3 axial, odd-parity equations decouple from the 7 polar, even-parity equations. Since the density and pressure perturbations (3.32) only contribute to the polar sector, we restrict to the 7 polar tensor harmonics in the following. A further simplification is obtained by choosing a convenient gauge. In the so-called Regge-Wheeler gauge [8] the 4 tensor harmonics (3.26-27) contribute only ([4], Table I). Then for $L \geq 2$ the metric perturbation is of the form ([4], equ. before (D5))

$$
h_{\mu \nu}=\left(\begin{array}{cccc}
-H_{0} & \sqrt{T_{L} / R-1} H_{1} & 0 & 0 \\
\sqrt{T_{L} / R-1} H_{1} & \left(1-T_{L} / R\right) H_{2} & 0 & 0 \\
0 & 0 & R^{2} K & 0 \\
0 & 0 & 0 & R^{2} K \sin ^{2} \vartheta
\end{array}\right) Y_{L}^{M}(\vartheta . \phi)
$$

where the functions in the matrix depend on $S$ and $R$ only.

Now from the non-diagonal elements of equation (4.1) one obtains the following four homogeneous partial differential equations ([4], equ.(C7a-f)):

$$
\begin{gathered}
\frac{\partial}{\partial S}\left(\frac{\partial K}{\partial R}+\frac{1}{R}\left(K-H_{2}\right)-\frac{T_{L}}{2 R\left(R-T_{L}\right)} K\right)-\frac{L(L+1)}{2 R^{2}} H_{1}=0 \\
\frac{\partial}{\partial R}\left[\left(1-\frac{T_{L}}{R}\right) H_{1}\right]-\frac{\partial}{\partial S}\left(H_{2}+K\right)=0 \\
-\frac{\partial H_{1}}{\partial S}+\left(1-\frac{T_{L}}{R}\right) \frac{\partial}{\partial R}\left(H_{0}-K\right)+\frac{T_{L}}{R^{2}} H_{0}+\frac{1-T_{L} / 2 R}{R}\left(H_{2}-H_{0}\right)=0 \\
\frac{1}{2}\left(H_{0}-H_{2}\right)=0 .
\end{gathered}
$$

Equation (4.5) holds for $\AA$ Ć $\geq 1$ only, The case $L=0$ requires a special treatment because $Y_{0}^{0}=$ const We return to this point at the end of the paper.. The remaining diagonal elements yield the following three equations with matter contributions:

$$
\begin{gathered}
\left(1-\frac{T_{L}}{R}\right)^{2} \frac{\partial^{2} K}{\partial R^{2}}+\left(1-\frac{T_{L}}{R}\right)\left(3-\frac{5 T_{L}}{2 R}\right) \frac{1}{R} \frac{\partial K}{\partial R}-\left(1-\frac{T_{L}}{R}\right)^{2} \frac{1}{R} \frac{\partial H_{2}}{\partial R}- \\
-\left(1-\frac{T_{L}}{R}\right) \frac{1}{R^{2}}\left(H_{2}-K\right)-\left(1-\frac{T_{L}}{R}\right) \frac{L(L+1)}{2 R^{2}}\left(H_{2}+K\right)=8 \pi G \delta p \\
\left(\frac{R}{R-T_{L}}\right)^{2} \frac{\partial^{2} K}{\partial S^{2}}-\frac{1-T_{L} / 2 R}{R-T_{L}} \frac{\partial K}{\partial R}-\frac{2}{R-T_{L}} \frac{\partial H_{1}}{\partial S}+\frac{1}{R} \frac{\partial H_{0}}{\partial R}+ \\
+\frac{1}{R\left(R-T_{L}\right)}\left(H_{2}-K\right)+\frac{L(L+1)}{2 R\left(R-T_{L}\right)}\left(K-H_{0}\right)=8 \pi G \delta \varrho \frac{R}{T_{L}-R}
\end{gathered}
$$




$$
\begin{gathered}
\frac{R}{T_{L}-R} \frac{\partial^{2} K}{\partial S^{2}}+\left(1-\frac{T_{L}}{R}\right) \frac{\partial^{2} K}{\partial R^{2}}+\left(1-\frac{T_{L}}{2 R}\right) \frac{2}{R} \frac{\partial K}{\partial R}+\frac{R}{T_{L}-R} \frac{\partial^{2} H_{2}}{\partial S^{2}}+ \\
+2 \frac{\partial^{2} H_{1}}{\partial R \partial S}-\left(1-\frac{T_{L}}{R}\right) \frac{\partial^{2} H_{0}}{\partial R^{2}}+\frac{2}{R-T_{L}}\left(1-\frac{T_{L}}{2 R}\right) \frac{\partial H_{1}}{\partial S}- \\
-\frac{1}{R}\left(1-\frac{T_{L}}{2 R}\right) \frac{\partial H_{2}}{\partial R}-\frac{1}{R}\left(1+\frac{T_{L}}{2 R}\right) \frac{\partial H_{0}}{\partial R}-\frac{L(L+1)}{2 R^{2}}\left(H_{2}-H_{0}\right)=8 \pi G R^{2} \delta p
\end{gathered}
$$

We have 7 equations for the 4 unknown functions $H_{0}, H_{1}, H_{2}$ and $K$ and for $\delta \varrho$ and $\delta p$. Since $S$ only appears in derivatives we go over to Fourier transformed quantities

$$
\hat{f}(q, R)=(2 \pi)^{-1 / 2} \int f(S, R) e^{i q S} d S,
$$

which are denoted by the same symbols without hat in the following. Equation (4.6) allows to eliminate $H_{0}$. Next Zerilli solves (4.3-5) for the derivatives of $H_{1}, H_{2}$ and $K$ (his equ.(F3a-c)):

$$
\begin{gathered}
\frac{d K}{d R}=\left(1-\frac{3 T_{L}}{2 R}\right) \frac{1}{T_{L}-R} K+\frac{1}{R} H_{2}-\frac{L(L+1)}{2 i q R^{2}} H_{1} \\
\frac{d H_{2}}{d R}=\left(1-\frac{3 T_{L}}{2 R}\right) \frac{1}{T_{L}-R} K+\left(1-\frac{2 T_{L}}{R}\right) \frac{1}{R-T_{L}} H_{2}-\left[\frac{i q R}{R-T_{L}}+\frac{L(L+1)}{2 i q R^{2}}\right] H_{1} \\
\frac{d H_{1}}{d R}=\frac{i q R}{T_{L}-R}\left(K+H_{2}\right)+\frac{T_{L}}{R} \frac{1}{T_{L}-R} H_{1} .
\end{gathered}
$$

If one substitutes these into (4.7), the left-hand side is zero. The same conclusion follows from (4.9). Consequently, the pressure perturbation $\delta p$ vanishes. But from (4.8) we see that the density perturbation is different from zero equal to ([4] equ.(F4))

$$
\begin{aligned}
16 \pi G R^{2} \delta \varrho=\left[\frac{3 T_{L}}{R}+(L-1)(L+2)\right] H_{2}- & {\left[(L-1)(L+2)+\frac{2 q^{2} R^{3}}{T_{L}-R}+\left(1-\frac{3 T_{L}}{2 R}\right) \frac{T_{L}}{R-T_{L}}\right] K-} \\
- & {\left[2 i q R+L(L+1) \frac{T_{L}}{2 i q R^{2}}\right] H_{1} . }
\end{aligned}
$$

Here the evolution equations (4.11-13) have been inserted. There remains to solve the equations (4.11-13) for $H_{1}, H_{2}$ and $K$.

\section{Evolution of the Perturbations}

From now on we deviate from Zerilli. Zerilli has combined the three first-order equations (4.11-13) with (4.14) to get one second-order equation for one single function. But this second-order equation cannot be reduced to quadratures. Instead we shall solve the linear system (4.11-13) in suitable variables and use the condition (4.14) to select the physical solution for the metric perturbations and to determine the density.

First let us study the density perturbation $\delta \varrho$. We consider energy-momentum conservation in the unperturbed metric

$$
\nabla^{\mu} t_{\mu \nu}=0
$$

Using (3.14) and linearizing about the vacuum background we have

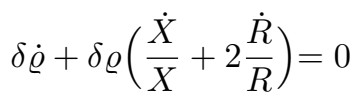

because the pressure vanishes. Here the covariant derivative in (5.1) was carried out in comoving coordinates by means of the Christoffel symbols [1], the dot means partial derivative with respect to comoving time. This gives

$$
\frac{\delta \dot{\varrho}}{\delta \varrho}=-\frac{\partial}{\partial t} \log \left(|X| R^{2} \hat{A} \check{s}\right)
$$


which can immediately be integrated

$$
\delta \varrho(t, r)=\frac{\varrho_{0}(r)}{|X| R^{2}}
$$

where $\varrho_{0}(r)$ is an arbitrary function of the comoving radial coordinate. With our results $(2.2)$ and $(2.7)$ with $Q=1$ we obtain

$$
\delta \varrho=T_{L}^{2} \frac{\varrho_{0}(r)}{\left|\sin ^{3} w\right| \cos w} .
$$

This shows that the energy density has a singularity at the Big Bang $w=\pi / 2$ and a strong one at the end $w=\pi$.

Instead of the Schwarzschild variable $R$ we shall use the redshift $z(2.8)$ in the following

$$
1+z=\frac{1}{\alpha|\cot w|}
$$

or rather

We then have

$$
x \equiv \alpha(1+z)=|\tan w|
$$

$$
\begin{gathered}
X=|\cot w|=\frac{1}{x} \\
R=T_{L} \sin ^{2} w=T_{L} \frac{x^{2}}{x^{2}+1} \\
T_{L}-R=\frac{T_{L}}{x^{2}+1}
\end{gathered}
$$

and

$$
\frac{d}{d R}=\frac{\left(x^{2}+1\right)^{2}}{2 T_{L} x} \frac{d}{d x} .
$$

Now the left-hand side of (4.14) becomes very simple

$$
16 \pi G R^{2} \delta \varrho=16 \pi G \frac{\varrho_{0}}{|X|}=16 \pi G \varrho_{0} x
$$

due to (5.3). Consequently it is good enough to solve the evolution equations (4.11-13) by power series in $x$. However, we shall see that (5.11) gives the density $\delta \varrho$ to leading order only, because the higher orders (4.14) are not contained in (5.2).

In the new variable $x$ our linear system has the following form

$$
\begin{gathered}
K^{\prime}=-\frac{x}{x^{2}+1}\left(1+\frac{3}{x^{2}}\right) K+\frac{2 H_{2}}{x\left(x^{2}+1\right)}-\frac{L(L+1)}{T_{L} x^{3}} H_{3} \\
H_{2}^{\prime}=-\frac{x}{x^{2}+1}\left(1+\frac{3}{x^{2}}\right) K+\frac{2}{x^{2}+1}\left(x+\frac{2}{x}\right) H_{2}- \\
-\left[q^{2} T_{L}^{2} \frac{2 x^{3}}{\left(x^{2}+1\right)^{2}}+\frac{L(L+1)}{x^{3}}\right] \frac{H_{3}}{T_{L}}
\end{gathered}
$$

and

$$
H_{3}^{\prime}=\frac{2 T_{L} x^{3}}{\left(x^{2}+1\right)^{2}}\left(K+H_{2}\right)+\frac{2}{x} H_{3} .
$$

Here the prime is the derivative with respect to $x$ and we have introduced the function

$$
H_{3}=\frac{H_{1}}{i q}
$$

in order to have real quantities only. The imaginary nature of $H_{1}$ comes from the $i$ in the definition (3.13) of the tensor harmonic $a_{L M}^{(1)}$. We also remove $T_{L}$ by the additional substitution

$$
H_{4}=\frac{H_{3}}{T_{L}}=\frac{H_{1}}{i q T_{L}} .
$$


By multiplying the three equations by suitable factors we obtain the following equations without fractions

$$
\begin{gathered}
\left(x^{5}+x^{3}\right) K^{\prime}=-\left(x^{4}+3 x^{2}\right) K+2 x^{2} H_{2}-L(L+1)\left(x^{2}+1\right) H_{4} \\
\left(x^{7}+2 x^{5}+x^{3}\right) H_{2}^{\prime}=-\left(x^{6}+4 x^{4}+3 x^{2}\right) K+\left(2 x^{6}+6 x^{4}+4 x^{2}\right) H_{2}-\left[Q^{2} x^{6}+L(L+1)\left(x^{4}+2 x^{2}+1\right)\right] H_{4} \\
\left(x^{5}+2 x^{3}+x\right) H_{4}^{\prime}=2 x^{4}\left(K+H_{2}\right)+2\left(x^{4}+2 x^{2}+1\right) H_{4} .
\end{gathered}
$$

Here we have introduced the abbreviation

$$
Q^{2}=2 q^{2} T_{L}^{2}
$$

The equation (4.14) for the density now reads as follows

$$
\begin{gathered}
16 \pi G R^{2} \delta \varrho=H_{2}\left(3+\frac{3}{x^{2}}+(L-1)(L+2)\right)-K\left[(L-1)(L+2)-2+\frac{Q^{2} x^{6}}{\left(x^{2}+1\right)^{2}}+\left(x^{2}+1\right)\left(\frac{1}{2}+\frac{3}{2 x^{2}}\right)\right]- \\
+H_{4}\left[\frac{Q^{2} x^{2}}{x^{2}+1}-L(L+1) \frac{\left(x^{2}+1\right)^{2}}{x^{4}}\right] .
\end{gathered}
$$

The system (5.17-19) has three linear independent fundamental solutions and the general solution is a linear combination of them. Since the perturbations are assumed to be small we must find the bounded solutions. A glance to (5.21) shows that these physical solutions have the following power series

$$
\begin{gathered}
K=\frac{a_{1}}{x}+\frac{a_{2}}{x^{3}}+\frac{a_{3}}{x^{5}}+\ldots \\
H_{2}=\frac{b_{1}}{x}+\frac{b_{2}}{x^{3}}+\ldots \quad H_{4}=\frac{c_{1}}{x}+\frac{c_{2}}{x^{3}}+\ldots
\end{gathered}
$$

This shows that the metric perturbations go to zero if the Big Bang $z \rightarrow \infty$ or $x \rightarrow \infty$ is approached. Substituting this into (5.17-19) and comparing the coefficients we obtain

$$
\begin{gathered}
a_{2}=a_{1}\left(2-4 \frac{L(L+1)+3}{2 Q^{2}+9}\right) \\
b_{1}=a_{1}\left(\frac{12}{2 Q^{2}+9}-1\right) \\
b_{2}=a_{1}\left(10 \frac{L(L+1)+21}{2 Q^{2}+25}-6 \frac{L(L+1)+9}{2 Q^{2}+9}-2\right) \\
c_{1}=-\frac{8 a_{1}}{2 Q^{2}+9} \\
c_{2}=4 a_{1}\left(\frac{L(L+1)+9}{2 Q^{2}+9}-\frac{L(L+1)+21}{2 Q^{2}+25}\right) .
\end{gathered}
$$

Here $a_{1}$ is a free overall factor, all other coefficients are fixed. The factor $a_{1}$ can also be fixed by substituting into (5.21). From the leading order $O(x)$ we find

$$
16 \pi G \varrho_{0}=-a_{1}\left(Q^{2}+\frac{1}{2}\right)
$$

so that $a_{1}(q)$ is determined by the $q$-dependence of the initial energy density $\varrho_{0}(q)$. From $(5.21)$ we then get the higher order perturbations of the density

$$
\begin{aligned}
16 \pi G R^{2} \delta \varrho=-a_{1} x\left(Q^{2}+\frac{1}{2}\right) & +\frac{1}{x}\left[-a_{2}\left(Q^{2}+\frac{1}{2}\right)-a_{1}\left(L^{2}+L-2-2 Q^{2}\right)+b_{1}\left(L^{2}+L+1\right)+\right. \\
& \left.+c_{1}\left(Q^{2}-\frac{L(L+1)}{2}\right)\right]+O\left(\frac{1}{x^{3}}\right) .
\end{aligned}
$$

The $L$-dependence shows that the density is essentially anisotropic. However, there is no explicit $M$ dependence. That means a deviation from axial symmetry can only come from an $M$-dependence of the initial condition $a_{1}$ (primary anisotropies). 


\section{Discussion}

The most interesting feature of our first order perturbative solution is that no finite initial condition is needed. The vanishing of the metric perturbations at the Big Bang $x=\infty$ uniquely fixes the solution apart from normalization $a_{1}$. On the other hand the energy density becomes singular at the Big Bang due to the first term in (5.30). Then first order perturbation theory is not good enough, we have to go to second order. This is also necessary for calculating the pressure which vanishes in first order. For late times pressure-less anisotropic dust is a good approximation, but the early Universe seems to be much more complicated.

In standard cosmology one introduces the critical density

$$
\varrho_{\text {crit }}=\frac{3 H_{0}^{2}}{8 \pi G} .
$$

Then the factor on the left-hand side of (5.30) assumes the following form

$$
16 \pi G R^{2}=\frac{3}{2 \varrho_{\mathrm{crit}}} \frac{\left(\alpha^{2}+1\right)^{4}}{\alpha^{6}}\left(\frac{x^{2}}{x^{2}+1}\right)^{2}
$$

so that equ.(5.30) can be written as

$$
\left.\frac{\delta \varrho}{\varrho_{\text {crit }}}=\frac{2}{3} \frac{\alpha^{6}}{\left(\alpha^{2}+1\right)^{4}} \frac{\left(x^{2}+1\right)^{2}}{x^{3}}\right)\left|a_{1}\right|\left[Q^{2}+\frac{1}{2}+O\left(x^{-2}\right)\right] .
$$

The factor $\left|a_{1}\right|(Q)$ (5.29) determines the density fluctuations at the time of last scattering (Sachs-Wolfe effect). There is no reason to expect $\delta \varrho$ near $\varrho_{\text {crit. }}$. Since $\delta \varrho$ in $(4.14)$ is a Fourier transformed quantity depending on redshift $x=\alpha(1+z)$, it is directly related to the power spectral function $P(Q)$ which can be determined from galaxy surveys. This will be discussed in a later paper.

The metric perturbations calculated in sect.5 are the basis for computing the CMB anisotropies. In the Gerlach and Sengupta's notation [6] we have to identify

$$
\begin{gathered}
h_{00}=-\frac{1}{x^{2}} H_{2 L M}, \quad h_{01}=H_{1 L M} \\
h_{11}=-x^{2} H_{2 L M}, \quad r^{2} K=T_{L}^{2}\left(\frac{x^{2}}{x^{2}+1}\right)^{2} K_{L M} .
\end{gathered}
$$

Then the integrated Sachs-Wolfe effect can be calculated by a formula due to Tomita [7]. This will be done in a later paper. Before this a basic question has to be discussed: How is the main isotropic part of CMB represented in our model ? It is important to note that until now we have excluded the spherical perturbation $L=0$. This requires a separate treatment which will be given in a forthcoming paper. By solving Maxwell equations in the nonstandard background we find that the $L=0$ perturbation indeed represents CMB. So nonstandard cosmology gives a very natural explanation why radiation is isotropic, but matter is not and is represented by pressureless dust.

Finally we should answer the question what is the small parameter which measures the smallness of $h_{\mu \nu}$ - a basic ingredient of every perturbation theory. As such one can take the inverse redshift $1 / z$ or $1 / x=X$.

\section{References}

1. Scharf G. 2015, Cosmology with the cosmic rest frame, arXiv astro-phys/1503.05878

2. Scharf G.2016, Gauge Field Theories, Spin 1 and Spin 2, 100 Years After General Relativity, Dover Publications, Inc. Mineola, New York

3. Plebański J. Krasiński A. 2006, An Introduction to General Relativity and Cosmology, Cambridge University Press

4. Zerilli F.J. 1970, Physical Review D 3, 2141

5. Bondi H. 1947, Spherically symmetrical models in general relativity, Mon. Not. Roy. Astr. Soc. 107, 410 
6. Gerlach U.H., Sengupta U.K. 1980, Physical Review D 6, 1300

7. Tomita K. 2010, Physical Review D 81, 063509, arXiv astro-ph/0912.4773

8. Regge T., Wheeler J.A. 1957, Physical Review 108, 1063

9. Krisciunas K. 2008, Type Ia Supernovae and the Acceleration of the Universe: Results from the ESSENCE Supernova Survey, arXiv:0809.2612 